\title{
Strates
}

STRATES Matériaux pour la recherche en sciences sociales

$11 \mid 2004$

Jeune recherche, la vitalité d'un laboratoire

\section{Habiter le dedans et le dehors : la maison ou l'Eden rêvé et recréé}

Nicole Mathieu, Annabelle Morel-Brochet, Nathalie Blanc, Philippe GAJEWSKI, Lucile Grésillon, Florent Hebert, Wandrille Hucy et Richard Raymond

\section{OpenEdition}

12 Journals

Édition électronique

URL : https://journals.openedition.org/strates/430

DOI : $10.4000 /$ strates.430

ISSN : $1777-5442$

Éditeur

Laboratoire Ladyss

Édition imprimée

Date de publication : 1 janvier 2004

ISSN : 0768-8067

Référence électronique

Nicole Mathieu, Annabelle Morel-Brochet, Nathalie Blanc, Philippe GAJEWSKI, Lucile Grésillon, Florent Hebert, Wandrille Hucy et Richard Raymond, « Habiter le dedans et le dehors : la maison ou l'Eden rêvé et recréé », Strates [En ligne], 11 | 2004, mis en ligne le 14 janvier 2005, consulté le 21 septembre 2021. URL : http://journals.openedition.org/strates/430 ; DOI : https://doi.org/10.4000/strates.430

Ce document a été généré automatiquement le 21 septembre 2021.

Tous droits réservés 


\title{
Habiter le dedans et le dehors : la maison ou l'Eden rêvé et recréé
}

\author{
Nicole Mathieu, Annabelle Morel-Brochet, Nathalie Blanc, Philippe \\ GAJEWSKI, Lucile Grésillon, Florent Hebert, Wandrille Hucy et Richard \\ Raymond
}

1 Cet article, élaboré à l'occasion du colloque «Espaces domestiques » organisé par Béatrice Collignon et Jean-François Staszak les 17-20 septembre 2002 à l'Institut de Géographie, est publié ici tel qu'il a été rédigé en janvier 2003. Pour en comprendre le sens, il est important d'avertir le lecteur qu'il répondait à un double objectif. D'une part, celui d'introduire une controverse au sein de ce colloque par la critique du terme «Espaces domestiques » proposé dans le texte d'appel à communications : il ne suffit pas d'accoler espace à tout qualificatif pour produire un concept géographique et son assimilation sans justification à la "dimension spatiale de la maison» réduit la «domestication » de l'espace à la relation corporelle et mentale au «dedans » alors que la tension entre l'appropriation du dedans et du « dehors » est vraisemblablement une tendance majeure de l'habiter contemporain. D'autre part, celui d'engager au sein du Ladyss un processus de confrontation et d'évaluation des expériences de recherche des jeunes chercheurs convoquant la question des rapports à la nature et aux lieux de vie des individus « ordinaires » dans le but de construire un groupe de travail qui interroge le concept de mode d'habiter comme évaluateur des rapports des individus et des groupes sociaux à leurs lieux et milieux de vie; ceci pour identifier à la fois les « bonnes pratiques » des lieux au regard de leur durabilité, et les « lieux et milieux favorables » à une habitabilité durable. Cette double motivation à laquelle s'ajoute le péril de l'écriture collective lui confère une qualité exploratoire dans un cheminement collectif qui ne semble pas encore abouti. Depuis, ce texte est une des bases du travail du Collectif "Mode d'habiter", aujourd'hui constitué, les commentaires en sa marge illustrent les premières pistes qui orientent vers une nouvelle rédaction théoriquement plus achevée.

2 Quand peut-on qualifier un espace de domestique ? Comment passe-t-on du domus au domestique et réciproquement? La «maison» est-elle toujours synonyme d'espace privé ? Être chez-soi ne renvoie-t-il qu'à l'intimité, au-dedans? Quelles relations 
doivent exister entre le dedans et le dehors, entre l'artificiel et le naturel, entre le fermé et l'ouvert, pour que l'espace soit habité dans le bien-être, pour qu'on se le représente et qu'on le pratique comme un chez-soi?

L'ambition de répondre collectivement à ces questions tient au fait qu'au sein du Ladyss, un groupe de chercheurs teste la validité du concept de mode d'habiter, à michemin entre celui géographique de genre de vie, et celui sociologique de mode de vie, pour son éventuelle pertinence dans l'observation et la théorisation de la façon dont les sociétés post-industrielles habitent (au sens fort) leurs territoires. Selon quel gradient dans les valeurs et les pratiques, et avec quelle conscience de la durabilité de leurs pratiques sur la durabilité des lieux, ces sociétés sont en interaction avec leurs milieux de vie, comme une espèce l'est avec son habitat? Deux paradigmes de la relation sociétés/milieux sont privilégiés. Le premier est l'investigation du point de vue de l'habitant, à partir duquel il faut tenter de définir les "cultures de la nature ", les représentations de la valeur des lieux, les catégories par lesquelles chaque sujet pense sa " géographicité ». Le second est la reprise des catégories de rural et d'urbain dont la confrontation est susceptible de renvoyer à des différenciations " générales " portant sur la qualité et les propriétés des milieux dans la mesure où elles permettent de placer (au sens propre) la relation des habitants à des espaces-temps différenciés, ayant du sens quel que soit le pays d'Europe concerné.

Dans cette problématique, la maison (l'espace domestique ?), tient une place essentielle puisqu'elle est le lieu habité par excellence mentalement et matériellement, au point qu'elle constitue le cœur de l'idée d'habitat. Dès le départ de l'exploration du concept de mode d'habiter (Mathieu, 1996), l'hypothèse fut faite du rôle joué dans la production d'espace et les pratiques de localisation résidentielle par le rêve (le modèle) de la maison rurale, avec ses propriétés. Son dedans - unifiant lieu de travail et de résidence -, et son dehors - au contact de la «nature» des saisons et de l'air "pur» sont antithétiques de l'appartement de centre ville ou de "cité ", au dedans petit, bruyant, aux formes imposées et stéréotypées, au dehors vicié, pollué, sans contact possible avec la «nature » mais aussi sans « intimité ». D'où l'investigation des représentations antiurbaines comme matrice des pratiques résidentielles.

Depuis ces premières pistes, il a été entrepris d'approfondir en interdisciplinarité (avec des architectes, physiciens, biogéographes, agronomes et anthropologues) ces nouvelles façons d'habiter ainsi que les décalages qui s'instaurent entre le rêvé et le réel, entre l'idéel et le matériel au sein de chaque habitant, de chaque famille habitante. Ces investigations commencent au cœur de ce qui a le plus de valeur de domus, de chezsoi, dans un mode de vie où la mobilité et la multi appartenance aux lieux deviennent la règle (sauf pour les personnes « captives » ou assignées à un seul lieu de vie).

6 Bien que leurs thématiques et leurs terrains diffèrent, chaque membre du collectif1 a entrepris une série d'enquêtes que nous appelons des «biographies résidentielles » ou des « récits de lieux de vie » et qui consistent à faire émerger dans un entretien long semi directif (souvent réalisé en plusieurs fois), les valeurs qu'accordent les enquêtés à tous les lieux qu'ils habitent ${ }^{2}$.

7 Le colloque est l'occasion de rassembler ces matériaux rendus disparates par les différences d'objectifs, de sites d'observation et de formes d'habitat et de confronter ce qui y est dit de la "maison », du "chez-soi », du dedans et du dehors, du privé et du public, de la nature et du vivant, jusqu'à dégager les points communs et généraux qui définissent un « territoire premier ». 
Chercher le général dans les modes d'habiter singuliers et les figures archétypales

Parmi les entretiens effectués, certains se détachent par le caractère exceptionnel du modèle architectural censé faire "maison », ou par la cohérence, parfois insolite, des discours et des pratiques des enquêtés. Malgré leur singularité, ces lieux de vie et le récit qui en est fait conduisent irrésistiblement le chercheur à faire l'hypothèse qu'il est en présence d'un "modèle" d'habiter dont il pourrait tirer des leçons de portée générale.

Planche 1 : une famille en toutes ses maisons

Habiter un passage à niveau : un modèle masculin rêvé de l'extérieur?

[Il se situe presque exclusivement en milieu rural]

10 Que peut nous apprendre la figure archétypale de la maison de garde-barrière et l'architecture du passage à niveau sur la question de la domesticité ? L'intérêt pour cette forme de " paysage ordinaire » vient du fait de son caractère paradoxal (Hébert, en cours). Habitat éminemment fonctionnel, il impliquait pour ses occupants, soumis au règlement de la $\mathrm{SNCF}$, que la liberté et le plaisir domestique s'effacent au profit du devoir et de ses charges. En ce sens, par sa disposition extérieure comme intérieure, il est l'archétype de la double captivité du garde-barrière et de sa demeure, en somme d'un espace "domestiqué ». Et pourtant, cet espace a toujours été et reste encore, l'Eden perdu, pour le petit garçon et l'amateur de modélisme. Depuis l'abandon des passages à niveau manuels, les maisons sont louées ou vendues, traduisant la persistance de l'engouement pour ce modèle réel et rêvé. Qui sont donc les habitants de ces lieux de vie contraints? Parviennent-ils toujours à contourner la forme porteuse de servitude, à définir l'intérieur intime et autonome d'un extérieur marqué par la règle et la dépendance? Ce modèle d'habiter est-il un espace domestique masculin, l'Eden perdu qui vit encore à l'état sauvage et qui reste à domestiquer ?3

Un modèle féminin ? La maison ronde ou circuler librement entre le dedans et le dehors

[Il se déploie aussi bien en milieu rural que dans des milieux urbains] d'intellectuels depuis les années cinquante jusqu'à aujourd'hui ? Ceci malgré la variété de leur taille, de leur ancienneté, de leur localisation, et donc du rapport entre le dedans et le dehors (Planche 1). Quelles propriétés ont-ils pour qu'ils aient tous été désignés par le terme de "maison » par chacun de leurs habitants, pour qu'ils aient été associés au "bien-être", voire à l'harmonie (le bonheur individuel et collectif)? L'exemple d'une famille, "heureuse en tous ses habitats " peut aider à théoriser les conditions objectives et subjectives qui font exprimer à quelqu'un qu'il habite un " endroit» comme un chez lui, comme une "maison", à repérer ce qui fait d'un « logement » un lieu habitable, un Eden, un « habitat familial » proprement dit ${ }^{4}$.

Propriété 1: l'intérieur, centre d'un cercle. Ce qui importe n'est pas tant la surface totale, le nombre et la taille des pièces, que le fait d'une disposition « ronde » donnant le sentiment que, de quelque point de l'intérieur que ce soit, existe la possibilité de se sentir au centre de l'ensemble, de pouvoir «circuler » aisément entre les lieux plus spécialisés ( $C f$. Planche 1, photos de l'appartement à la Bastille et le studio à Paris) que sont les lieux de «travail » (coins des devoirs ou de la musique pour les enfants; où l'on a son bureau pour les adultes actifs; où l'on exerce l'économie domestique), et ceux où 
l'on se repose (réside, rest), où l'on se recrée en y passant le temps libre (non contraint) que l'on appelle loisir (paresse, leisure), qui sont marqués par des meubles particuliers (fauteuils, sofas, coussins, cosys et évidemment lits). Surtout du point de vue féminin, la «bonne» circulation est une propriété essentielle pour que les habitants d'un intérieur, quels que soient leur âge et leur statut, le désigne comme un chez-soi, comme une " maison » où chacun a sa place dans l'ensemble, comme un " espace domestique " ayant une valeur matérielle et idéelle harmonieusement combinée.

Mais, pour qu'il y ait bien-être, l'intérieur doit aussi être disposé de telle sorte qu'il donne le sentiment à l'étranger, à l'hôte ( $C f$. Planche 1, photo Cilof à Viry), celui qui ne fait pas partie du premier cercle de ceux qui habitent l'endroit, qu'il est accueilli. Lui aussi doit pouvoir circuler librement d'un endroit à l'autre sans réserve, mais avec l'évidence qu'existe une hiérarchie sensible mais discrète entre les lieux, en fonction de leur plus ou moins grande intimité, ce qui n'exclut pas le partage. D'où l'importance de la première pièce (celle par où l'on entre même s'il n'y en a qu'une), qui doit exprimer symboliquement et matériellement l'ouverture aux autres et l'absence de coupure interne (mis à part le lieu de la totale intimité où l'on se lave et où l'on fait ses besoins).

Pour conforter cette double propriété, toute la disposition du dedans s'oriente par rapport à la lumière et aux fenêtres, donc par rapport au dehors.

Propriété 2 : intégrer la discontinuité du dehors. Pour être bien chez-soi, les propriétés de l'autour sont essentielles. Que la "maison » soit située en ville ou à la campagne, en surplomb d'une place ou d'un jardin potager ( $C f$. Planche 1, photo Bastille, maison rurale), l'important est que l'autour permette d'affronter la discontinuité entre le dedans et le dehors, d'intégrer celui-ci à l'espace rond, de s'approprier visuellement et affectivement les abords. Certes, plus cet abord est minéralisé, artificialisé, marqué par des arrêts (escalier, porte étroite fermée par un code etc.), plus la distinction dedans/ dehors est accentuée. Mais pour compenser cette rupture, un certain nombre d'endroits proches deviennent alors les appendices affectifs de la maison: le café d'en face où l'on peut laisser sa clé et des messages pour famille et étrangers de passage, le marché du dimanche matin (qui est l'équivalent d'une balade en forêt ou à la campagne dans un rapport non seulement avec les « voisins » mais aussi avec le vivant), le métro et les stations d'autobus parce qu'ils permettent d'accéder au monde extérieur (lieux de travail, de loisirs hors de la maison, de rencontres avec les autres dans d'autres " maisons », de vacances ou de résidence récréative).

17 La catégorie de « chez-soi » apparaît pleinement lorsque la rupture, toujours objective, entre le dedans et le dehors, est atténuée par l'appropriation (intégration au sein du domestique) de l'abord, dans un double mouvement du regard sur la maison et du passage aisé du dedans au dehors. En ce sens la maison individuelle et le rez-dechaussée sont les plus porteurs de valeurs. Le petit jardin en ville donne un sentiment équivalent à la maison rurale d'où l'on voit le jardin potager de plus loin, la petite maison, les arbres plantés aux différentes dates connues. Sans jardin autour ou à proximité, le sentiment de continuité entre le dedans et le dehors se construit autrement et par extension de l'espace habité : l'arbre sur fond de ciel, les couleurs et les formes du vis-à-vis, mais aussi l'espace des pratiques (de travail, de circulation et de recréation) deviennent le chez-soi. Si la disposition de la «maison» exclut ce sentiment, se développe alors, à l'intérieur, des pratiques qui reconstituent cette perméabilité entre deux milieux distincts (dedans et dehors) soit par une terrasse, soit 
par un balcon, soit si cela est absolument impossible par la mémoire (toujours présente) de l'autre lieu («l'autre maison») où ce rapport est à la fois rêvé et pratiqué .

La nourrisseuse ou l'animal révélateur du besoin d'étendre l'espace de l'intimité audelà du domestique animaux, mais celui que je préfère, c'est le chat car il correspond à mon caractère. » ou encore «Voyez, j'ai des rideaux, et je ne les tire jamais : j'ai été élevée dans la rue, moi!». Ce mode de vie implique la négation d'une rupture entre l'intérieur et l'extérieur au nom du rapport à la nature : «J'aime la nature et je ne me verrais pas enfermée. La nature, c'est le dehors, ce n'est pas être enfermée. ». Cette vision d'un chez-soi étendu se conjugue ici avec une vision historique et édénique de l'espace urbain. Autrefois " une végétation dense accueillait chats et oiseaux vivant en bonne harmonie avec enfant et adultes. " Cette idée d'une harmonie, d'une continuité des espaces et des espèces se rapproche, sur le plan intellectuel, de certaines utopies urbaines.

Cette femme propose donc une vision et un usage de l'espace originaux qui s'expriment à travers une appropriation symbolique et matérielle, une transformation de l'espace urbain, au même titre que les tags. Or, quelles que soient les villes étudiées, les citadins ont le plus souvent une faible marge d'action, l'appropriation des espaces se réduit généralement à des façons de les utiliser ou de les voir. Pourtant, l'idée que l'urbanisme doit favoriser la sociabilité locale et la continuité entre espace de l'intime et espace extérieur (public) se développe. L'habitant doit pouvoir habiter au-delà de son espace domestique, se sentir chez lui dehors. Certes le citadin moyen ne transforme pas 
l'espace public, mais les nourrisseurs, eux, contribuent par leurs pratiques, à la production de milieux urbains. La loi du ${ }^{6}$ janvier 1999 (loi 99-5, JO p. 329) qui reconnaît ce type de rôle social qualifié de gestion déléguée inaugure peut-être la prise en compte d'une demande sociale de qualité de vie en ville et de la nécessaire appropriation responsable de «l'extérieur ».

Notre «nourrisseuse » fait ainsi de l'espace urbain un chez-soi, conforme à son idée de la relation intime entre l'homme et l'animal car elle cherche à réinstaurer un espace où ils partagent l'Eden urbain, une sur-nature urbaine, sorte de jardin où les espaces de la cohabitation ont été recréés?

Comprendre le rapport au dehors pour définir le chez-soi

Dans le collectif, l'entrée par l'autour (l'environnement) des habitants urbains ou ruraux est privilégiée par certains pour interroger les modes d'habiter. Alors que les recherches précédentes partent du cœur de la résidence, du « feu » de l'habitant, cellesci s'y dirigent depuis le " dehors », l'espace dit « naturel », le quartier censé enchâsser le chez soi, l'espace public de la rue, de la place voire du café. C'est du point de vue de l'extérieur que sont interrogées, dans la diversité des cohabitations, les représentations et les pratiques des individus enquêtés.

La part naturelle de l'espace domestique

[L'analyse se situe en milieu rural]

Comment est identifiée la «nature » dans un espace habité ? Comment des agriculteurs, des résidents en maison individuelle (disposant d'un jardin petit ou vaste, ouvert ou non sur la rue), et des habitants d'immeubles collectifs, pensent et pratiquent le rapport entre ce qu'ils identifient comme nature et ce qu'ils définissent comme un «chez-soi »? La nature, qu'on dit domestique pour l'opposer à la sauvage, fait-elle partie de l'espace domestique? S'y oppose-t-elle ou contribue-t-elle à sa domestication? Les « récits de lieux de vie » menées dans le Vexin français (Raymond, 2004) apportent à ces questions des réponses originales.

Rejoignant les nombreuses analyses sur les rapports à la nature et aux paysages, les enquêtes confirment la valorisation générale de ce qui est identifié comme étant de la nature, qu'elle soit reconnue sauvage ou domestique (jardinée, cultivée...). Mais deux constats sont plus inattendus. D'une part, certains éléments constitutifs des espaces habités sont identifiés comme étant de la nature. D'autre part, cette nature est essentielle à la constitution du cadre de vie que chacun désire ou se recrée. Elle participe à l'identification de territoires que l'on reconnaît ou que l'on revendique habiter. Il convient alors de s'interroger sur la part de nature constitutive d'un « chezsoi » et de désigner les éléments matériels qui la composent, afin d'approcher l'étendue de ces territoires.

Lorsque les éléments de nature présents dans l'espace domestique (appartement) se limitent aux végétaux cultivés en pot, les personnes ont toutes un mode de vie majoritairement urbain et l'espace intérieur s'oppose fortement à un extérieur, à un dehors non approprié, à un espace urbanisé et dévalorisé.

S'il y a jardin, celui-ci étend l'espace de nature domestiquée hors du cadre bâti de la maison. S'il est soustrait aux regards des autres (clôture opaque, disposition à l'arrière de la maison), cet espace de nature est constitutif de l'espace de résidence. Il incarne le bien-être et légitime le choix de la résidence. Ces personnes ne recherchent alors que très peu la nature hors de leur jardin. 


$$
\text { dehors }
$$
Le dehors et le dedans s'y confondaient. Ces formes de sociabilité et d'usage de l'espace
ont quasiment disparu à présent, du fait de l'évolution d'une génération et de la réhabilitation du quartier qui a décuplé les loyers et contraint nombre d'habitants (artistes et artisans) à partir et à laisser la place à des bureaux. Un clivage s'est alors créé entre les anciens (en minorité) et les nouveaux car le Passage comme lieu unifiant travail et résidence n'existe plus. En conséquence, l'investissement des lieux communs (paliers, cours...) est devenu délicat, les territoires de sociabilité se sont réduits à l'escalier ou ont disparu... : autant de facteurs rendant plus brutales et moins perméables les limites entre le chez-soi, celui des autres et celui de tous. Enfin, si les lieux comportant de vastes espaces communs restent cependant valorisés, si être du Passage (habitant) reste un référent identitaire fort, c'est que disposer d'un dehors à la fois privé et collectif demeure essentiel à la constitution du «chez-soi ». C'est aussi parce que de tels espaces jouent d'autant plus le rôle d'espaces transitionnels, d'« espace d'adaptation » entre le dedans et le dehors (logement, palier, escalier, cour, passage, rue) que le rapport à la rue est vécu comme agressif. dehors. Le Passage est marqué par son histoire communautaire: "les locataires 
Planche 2 : Physique des lieux et lieux vécus : le quartier ancien de la Bastille quartier, ceux qui «ne sont pas à la mode », ceux où l'on peut avoir ses habitudes, sont les plus valorisés par les habitants, et ce, qu'ils y habitent depuis cinq ou trente ans, qu'ils soient étudiants, artistes ou chefs d'entreprise. Les changements physiques et l'augmentation de la fréquentation du quartier par une population consommatrice et non résidente sont vécus comme une détérioration du quartier et du bien-être à y vivre : « le quartier n'est plus fait pour ceux qui y vivent... c'est devenu un quartier fastfood, un quartier à consommer.» En effet, se sentir chez-soi en dehors du logement nécessite une "familiarité ", des repères, une interconnaissance que le mouvement constant des populations et des commerces altère. stigmatisés pour leur densité humaine et leur encombrement. La difficile circulation piétonne dans certaines rues, entraînant un sentiment d'étouffement, d'oppression, est un point déterminant du mal-être ressenti dans ces espaces. Mais cette gêne ancienne est devenue un problème quand les « étrangers au quartier » se sont faits omniprésents et que l'économie locale s'est entièrement tournée vers eux.

Autre stratégie : la désidentification verbale au quartier. On se disait « de la Bastille», on se dit maintenant « d'Aligre " par exemple, où « il reste encore une vie de quartier en dehors du marché le dimanche ».

Toutefois, parce que l'attachement au quartier ou au Passage reste fort, le délaissement des espaces centraux au profit des marges se traduit moins par un repli sur un dedans réduit au logement, que par une recherche (rêvée ou effective) d'une sociabilité et de pratiques édéniques passées. Ces comportements socio-spatiaux sont vécus comme des formes de résistance à une détérioration de la qualité sociale et physique du milieu de vie des habitants.

40 Une complexification du territoire de vie des habitants (à l'échelle du quartier) s'opère alors, avec des sous-ensembles identifiés et fortement hiérarchisés du point de vue idéel. Le territoire à vivre des habitants n'est pas réduit, mais sa morphologie change : il s'en trouve plus éclaté, plus discontinu, comme autant d'enclaves où ils prolongent leurs chez eux'.

Le café : espace public ?!

[L'analyse se situe presque exclusivement en milieu rural]

S'il a un statut d'espace commercial et public, le café répond parfaitement à la définition de l'espace domestique. Se sentir chez-soi, s'autoriser à ne plus interpréter l'endroit mais le vivre, y avoir ses habitudes, n'est pas l'apanage du logement. Les débits de boissons sont révélateurs d'autres territoires, spécifiques, premiers, sociaux (Gajewski, en cours).

Être à l'aise dans un café, c'est prendre possession du lieu, de la place, place souvent réduite à une table, à une chaise que le client fait siens. En quelques minutes, le périmètre pensé comme acquis augmente selon la densité de population environnante, la durée du séjour, le nombre de commandes passées et les discussions engagées. Tout mouvement physique dans le café élargit l'espace approprié. Le sentiment d'être à sa place, de légitimer sa position dans cet espace s'intensifie lorsque de nouveaux clients arrivent et reproduisent un schéma «d'installation» similaire. L'installation aboutit à la 
construction d'un espace d'intimité, associant l'individu seul à un groupe ou à un essaimage d'autres individus seuls.

Les habitués, ceux qui viennent quotidiennement retrouver «les copains » autour du zinc, ceux qui sont servis sans passer commande, qui ne connaissent plus les préliminaires d'appropriation, sont chez eux car leur présence participe à l'élaboration du groupe, à son identité rattachée au lieu; et la présence d'étrangers (au groupe) galvanise un comportement démonstratif d'une légitimation d'appartenance au groupe des habitués. Néanmoins il faut différencier les "habitués» des personnes ayant coutume d'aller au café. Ces derniers y entrent souvent sur rendez-vous et/ou en groupe et privilégient la salle. Le dédoublement observé du sentiment du chez-soi en dehors (au café) correspond en fait à la délimitation d'un espace refuge, choisi selon des critères d'affinité, qu'elle soit sociale ou spatiale. Il est un troisième endroit, un espace intermédiaire entre travail et maison, entre espace public et privé. Dans l'appropriation du lieu par les clients, il faut distinguer les différents espaces du café. Tout d'abord l'espace le plus intimiste, l'antre du débit de boissons : le bar. S'asseoir au comptoir, c'est se mettre à disposition sociale du patron et des autres clients perchés sur leur tabouret. La salle quant à elle est un espace intermédiaire entre le dedans et le dehors ; des habitués s'y rassemblent mais aussi des clients plus occasionnels qui rappellent aux habitués la présence du dehors, de l'englobant. La terrasse enfin est pour ainsi dire l'espace le plus commercial du débit de boissons. L'acte de consommer y est réduit à sa plus simple expression, les discussions s'effectuent entre personnes venues en groupe et obligent le patron à sortir de son café. Elle est l'espace extérieur, une extériorité commerciale, sociale, relationnelle, spatiale. Si deux cafés se jouxtent, le premier disposant d'une terrasse et de baies vitrées, le second ne disposant que d'une salle, les clients occasionnels privilégieront le premier.

Par ailleurs, le paradoxe du débit de boissons est son statut légal d'espace public. Un boulanger dans sa boulangerie est chez lui, un patron de café de l'est pas. Pourtant le sentiment d'être chez-soi est plus marqué chez le cafetier que chez les autres commerçants. Dans certains villages du nord Aveyron, le responsable du café n'est pas, dans les représentations du groupe villageois, un commerçant mais le gardien de la salle sociale du village. Lors de manifestations exceptionnelles, sur la place du village ou dans la salle des fêtes, le patron ferme son commerce à la demande collective car sa participation y est quasi obligatoire. Dans ces cafés communautaires, la clientèle villageoise y entre sans distinction d'âge, de sexe ou de classe sociale et les jeunes des villages alentour n'y entrent pas ; même si généralement les jeunes clients sont les plus mobiles et aptes à changer de café. Ils ne reconnaissent pas cette forme de communauté de village qui brasse les classes d'âge.

Ainsi, intuitivement se mettent en place des stratégies de communautés, faites de tentatives d'intégration ou d'exclusion, qui évoquent implicitement des structures mentales, des cadres géographiques et sociaux, des intérêts particuliers et parfois collectifs ${ }^{10}$. Dans un village communautaire, les limites du sentiment de «chez-soi » ne sont plus les maisons individuelles ni le café mais le pourtour du village, excluant de ce territoire les hameaux avoisinants. Le débit de boissons devient alors le lieu d'où émerge une identité locale délimitée par la morphologie du village.

En reprenant la définition de l'espace domestique, le café est le produit d'une société dont il porte les normes. En même temps, il structure la vie quotidienne et participe à la reproduction sociale. 
Vers la théorisation générale des rapports entre le chez-soi et les modes d'habiter

C'est à l'occasion de contrats de recherche inclus dans le programme «La nature dans la ville» du Ladyss que deux chercheurs ont tenté de construire une typologie des modes d'habiter (représentations et pratiques des rapports aux natures et aux matérialités urbaines) à partir de six sites ( $C f$. Planche 1 dans l'article de L. Grésillon p. 94 et 3 dans celui-ci) exemplaires des milieux de deux villes, Paris et Rouen. Ces typologies encore exploratoires, révèlent à la fois des convergences sur les processus par lesquels le «logement" devient un «chez-soi» mais aussi des contrastes. Renvoient-elles aux différences des points de vue des chercheurs ou à celles des milieux urbains concernés?

Habiter le dedans et le dehors à Paris : une question d'urbanité ?

Les entretiens menés sur Paris (Grésillon, en cours et article dans ce numéro) confirment l'extrême variété de l'usage et de l'étendue du chez-soi. Pour certains, il a une dimension locale "mon quartier", pour d'autres, il débute dans les espaces intermédiaires entre la rue et l'habitat: « quand je rentre dans le square, je suis chez moi ». Pour d'autres encore, il a les contours du logement ou même, il n'en est qu'une partie : « ma pièce à moi ». Aux représentations des frontières du chez-soi s'ajoutent les limites matérialisées par les pratiques. Les techniques pour protéger son logement de la pollution, en particulier rue Lagrange (planification journalière de l'ouverture des fenêtres ou imperméabilisation de l'habitat par des filtres dans les conduits de cheminées), au même titre que l'usage des balcons et jardins (ignorés, réservés exclusivement aux plantations ou investis pour un bain de soleil) illustrent la représentation des qualités biophysiques du chez-soi et la construction de ses contours. Si ces limites idéelles et pratiques ne concordent pas forcément, il n'y a pas d'incohérence si l'on considère que le chez-soi a plusieurs enveloppes représentatives des coquilles de l'individu (Moles, Rohmer, 1978), vecteur de son appropriation de l'espace. Seulement au temps « $t$ » de l'entretien et dans son contexte d'énonciation, le chez-soi est pour la personne interrogée une de ses coquilles plus qu'une autre. L'analyse des entretiens montre une certaine corrélation entre l'ampleur spatiale du chez-soi, la fonction qu'on lui donne, l'étendue et l'usage du territoire parisien parcouru fréquemment.

Ont pu ainsi être distingués trois manières d'investir l'habitat et de pratiquer la ville,

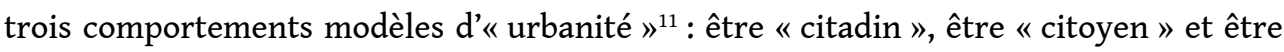
« habitant»:

51 - pour le «citadin», l'espace urbain ne vaut que pour les activités qu'il offre. Son périmètre d'investigation est à l'échelle de la capitale, mais il ne vit les lieux que s'ils sont le but de ses déplacements et qu'il s'y sent sécurisé : son bureau, sa maison ou celle de ses amis. Il ne parle pas des endroits qu'il fréquente en termes de sensation. Il se déplace essentiellement en voiture, ou décrit les temps de transport comme une parenthèse mentale et corporelle. Le logement est aménagé à son goût, il y a fait d'importants travaux et porte une attention extrême à la décoration. Il investit peu les balcons qu'il possède et ne montre pas d'intérêt marqué pour les plantes. Quand il a une maison de campagne, il semble y réserver l'activité de jardinage et le déploiement de ses sensations. Le chez-soi est réduit à sa plus stricte délimitation - les murs - et forme un véritable cocon, un Eden, conçu comme imperméable. Ce «citadin » vit le dedans et le dehors de manière opposée : la maison est conçue et habitée comme un havre alors que l'extérieur est perçu comme hostile. 
52 - le «citoyen » profite quant à lui des opportunités relationnelles et des nombreuses possibilités d'engagement que lui offre Paris. Cet individu utilise en majorité les transports en commun et ne s'en plaint pas. Ses déplacements sont très centrés autour du logement. Il évalue les lieux de la ville en fonction de leurs qualités de sociabilité et les fréquente à cette aune. L'aménagement intérieur importe peu: il y a peu de végétation parce que "ça prend du temps ", temps consacré en majorité aux activités extérieures. La porosité du logement par rapport à l'environnement biophysique et social n'est pas une préoccupation, le rapport entre le dehors et le dedans n'est pas ressenti comme devant être construit. L'habitat est un point d'ancrage autour duquel le « citoyen » gravite.

53 - enfin, l'attrait de la ville provient pour «l'habitant» de ses replis cachés, de ses revers campagnards, de ses espaces intimes inattendus : « les petites places, les petites rues, les jardins, les courettes [...] c'est la ville cachée dans la ville». Il parcourt Paris essentiellement à pied et par plaisir dans un rayon qui dépasse fréquemment son arrondissement. Il parle souvent de la capitale en terme sensible et décrit certains lieux, tout comme son habitat, par des odeurs, des sons, des couleurs. Il semble appliquer la même sensibilité partout, quelle que soit la raison de sa présence dans les endroits. La plupart du temps, il choisit son habitat pour son cadre de vie et possède des plantes. Il aime jardiner et la présence de végétation est importante. Il prend en compte la pollution en gérant le rapport entre le dehors et le dedans au niveau des interfaces (les fenêtres essentiellement) parce qu'il les considère en interrelation. A travers les plantes, le plaisir sensoriel de découvrir la ville, la préoccupation des conditions de vie (qualité de l'air, salubrité de l'eau), il montre qu'il conçoit le dedans et le dehors comme faisant partie d'un même système, la ville est un milieu.

Il semble, d'après ces modèles de personnalité urbaine, qu'il y ait une dépendance entre ce que l'individu attend de la ville, ce qu'il y puise et la manière d'y vivre le dedans et le dehors. Si l'espace urbain est un concentré d'opportunités fonctionnelles, seul son contenu - donc son dedans - est vécu. Si la ville ne vaut que pour ces possibilités relationnelles, le dehors - lieu des rencontres - est l'endroit de ressource. Lorsque la saveur de la ville vient de l'ensemble de ces caractéristiques biophysiques, alors le dehors et le dedans sont habités avec autant d'intensité, la limite entre ces deux espaces étant évanescente.

À Rouen, vers une réduction du chez-soi par l'autour

L'analyse des récits de lieux de vie dans les milieux intra-urbains de Rouen (Hucy, 2002) permet de distinguer quatre tendances fortes dans l'élaboration et surtout la reproduction des formes de l'espace domestique.

Planche 3 : les modes d'habiter l'agglomération rouennaise : trois sites

La première tendance est de faire de l'espace domestique un refuge contre un environnement extérieur considéré comme hostile. Ce type regroupe des personnes qui, en général, n'ont eu le libre choix ni de leur logement ni de sa localisation (HLM). Il y a tout d'abord un rejet de cette forme de logement qui, selon les cas, va s'étendre au quartier, aux autres habitants qui ne partagent pas le même mode de vie, et enfin, à la ville, considérée comme une source de maux. Du point de vue des pratiques, cela se traduit par une minimisation des rapports avec l'espace public englobant et la réduction de l'espace domestique au logement, voire à une seule pièce. Ce mode d'habiter implique moins une appropriation de cet espace que sa fermeture. 
La seconde est de faire de l'espace domestique un lieu fonctionnel. Alors, l'espace domestique est le lieu qui maximise les fonctionnalités de l'espace à vivre. Plutôt qu'un endroit privé, il est celui qui permet de vivre le mieux possible. Il s'apparente à tous les lieux du quotidien et plus seulement au logement. On doit ici parler d'espace vital. Plus prononcé chez la gent masculine, ce type de rapport concerne aussi des femmes. La définition des lieux passe donc par une appropriation fonctionnelle et non matérielle de l'espace.

L'espace domestique peut aussi être l'espace de l'intime. Cette modalité des rapports au chez-soi est caractéristique des femmes, propriétaires de leur logement, n'exerçant pas d'activité professionnelle. Bien qu'il s'agisse d'un achat en couple, ce sont elles qui ont présidé au choix de la maison ou de l'appartement. Elles ont par la suite entièrement reconfiguré l'intérieur, et l'extérieur dans la mesure du possible, à leurs goûts. Extrêmement structuré, cet espace est relativement ouvert sur le domaine public, ce dernier participant même de sa définition. Ici, le « chez-soi » est un espace intime mais aussi un espace social.

, et il est possible que cette tendance se développe, certains font de l'espace domestique un espace projeté et rêvé. Quand il y a multirésidence, le "chez-soi » est rarement associé à la résidence principale parce qu'elle est urbaine et synonyme de vie active. Les personnes projettent alors, dans leur résidence secondaire où dans un lieu qu'elles fréquentent assidûment à "l'extérieur ", les vertus supposées du lieu de vie idéal, de l'Eden. On est ici dans l'expression d'un phénomène similaire à la première tendance, bien que le rejet vise davantage l'espace urbain que la société. Ici le chez-soi est ailleurs.

0 L'espace domestique est donc produit par des stratégies individuelles, il est une construction personnelle. Un même espace peut accueillir l'espace domestique de plusieurs, sans qu'il s'agisse jamais du même. Chacun cherche à conformer sa représentation intime à la matérialité du lieu. Pourtant, au-delà de ce particularisme, se dessinent des profils identitaires remarquables ainsi que des propriétés générales qui font de l'espace « domestique » avant tout l'espace de la famille au sens de foyer, le lieu dans lequel chacun se reconnaît. "L'extérieur " se définit en fonction de ce qui est considéré comme l'espace public (qui appartient à tous et à personne), au-delà, c'est aussi le moyen d'affirmer ou de définir sa place dans la société (« localisation sociale »). De plus, le «chez-soi » a pour marqueur l'appropriation verbale («c'est ma ville, ma maison - même s'il s'agit d'un appartement -, mon commerçant ») ou physique, l'investissement personnel. C'est un lieu, un endroit, à fermer.

61 Analysées dans une perspective temporelle, les biographies résidentielles rouennaises tendent à montrer une contraction progressive de l'espace domestique, qui, ne pouvant plus être le « quartier ", se réduit à l'immeuble et le plus souvent à une ou deux pièces $\mathrm{du}$ logement. Ceci serait à mettre en parallèle avec la déliquescence des structures de l'espace public dont la désappropriation entraînerait celle de l'espace privé et intime. Dès lors la notion d'espace domestique n'est-elle pas peu adaptée à la multiplication des modes d'habiter les milieux de vie et aux difficultés à établir les frontières entre privé et public, intérieur et extérieur?

Conclusion

62 Il nous faut prendre acte des nombreux points de convergence émergeant de tous ces récits et de leur analyse. Tout d'abord l'idée du chez-soi, du bien-être dans l'habiter, repose sur un imaginaire, une «fiction » que plusieurs descriptions de " maisons » et 
d'« habitants » ont illustrés. Il y a bien de l'Eden dans la domestication de l'espace, et toujours un effort pour transformer le rêvé en pratiqué.

Ensuite, l'intimité, le socle du chez-soi, ne se réduit pas au dedans et au privé. Le besoin d'étendre l'espace familier (domestique) constitue une aspiration lourde qui se traduit par l'appropriation du dehors (ensemble d'espaces extérieurs choisis et pratiqués), qu'il s'agisse de quartier, de nature ou du café. Ni l'opposition entre le dehors et le dedans, ni celle du public et du privé ne permettent l'appréhension de l'espace de référence, de l'endroit où l'on demeure, de son «chez-soi ». Si l'espace domestique est un territoire premier, c'est-à-dire, par analogie aux nombres du même nom, un territoire indivisible, espace irréductible d'un "chez-soi ", alors il doit être étendu à l'espace qui contient l'ensemble des références constitutives de cette identité spatiale.

Enfin il existe des manières d'habiter, des types d'habitants et cette typologie ne tient pas qu'à la forme des "dedans" et des "dehors", mais au rapport entre les représentations et les valeurs des individus d'une part, et, d'autre part, la possibilité d'aménager, de s'approprier le dedans, et surtout le dehors, par des stratégies et des pratiques qui garantissent le prolongement de soi et de sa famille au-delà de l'habitat.

Le colloque nous a conduit à une réflexion sur les mots utilisés pour approcher, même en la contestant, la notion d'espace domestique. Réflexion sur les mots convergents (dedans/dehors, "territoire premier", chez-soi), les mots manquants chez certains (maison, nature) et présents chez d'autres (refuge, Eden, intimité), sur les notions controversées (espace domestique, domus, public/privé). Réflexion aussi sur la valeur du croisement des «études de cas» exemplaires et de la méthode typologique pour faire apparaitre les processus généraux qui définissent le territoire premier et son extension.

Notre effort de confrontation débouche aussi sur la remise en cause de certaines idées devenues stéréotypes : que l'habiter est une fonction séparée des «fonctions clés de l'urbanisme de la Charte d'Athènes (1933): habiter, travailler, se récréer, circuler ", alors qu'elle les contient toutes au moins dans l'idéel; que certains espaces (l'espace agricole ou rural) sont plus habitables, sous prétexte des racines paysannes de la culture française, de la présence de paysages ouverts et d'espaces naturels. Tous les lieux sont habitables s'ils sont pensés comme des «chez-soi ». La montée actuelle de la "question habitante» n'est-elle pas le refus de l'assignation à des espaces refuges entourés d'inhabitable? N'est-elle pas revendication d'une responsabilité accrue de chacun sur l'espace qu'il rêve et veut habiter?

\section{BIBLIOGRAPHIE}

La bibliographie est strictement limitée aux recherches des auteurs qui forment la base de cet article. Il est évident qu'un état des lieux des publications, d'ailleurs fort abondantes, dans lequel il s'inscrit est en cours de réalisation. Le Collectif Modes d'habiter organise un séminaire en 2004 autour des auteurs « fondateurs » comme « actuels » qui sont les plus à même de l'aider à 
consolider les hypothèses et la tentative de théorisation engagée par la problématique des nouveaux modes d'habiter en relation avec le rural et l'urbain.

Blanc N., 2000, Les animaux et la ville, Paris, Odile Jacob.

Gajewski P., (en cours), Le rôle du débit de boissons en milieu rural. Analyse comparée de régions de moyenne montagne en France et en Écosse, Paris, Université Paris 8.

Grésillon L., (en cours), Vivre la ville : odeurs et pratiques urbaines, Paris, Université Paris 1

Hébert F., (en cours), Les figures de la routine, Paris, Université Paris 1.

Hucy W., 2002, La nature dans la ville et les modes d'habiter l'espace urbain. Expérimentation sur l'agglomération rouennaise, Rouen, Université de Rouen.

Mathieu N., 1996, «Rural et urbain : unité et diversité dans les évolutions des modes d'habiter », in Jollivet M., Eizner N. (dir.), L'Europe et ses campagnes, Presses FNSP, p. 187-216.

Mathieu N., Pilleboue M., La maison ronde ou l'espace des femmes (en cours).

Moles A., Rohmer E, 1978, Psychologie de l'Espace, Paris, Casterman.

Morel-Brochet A., (en cours), Les modes d'habiter urbains et ruraux à travers la mobilité géographique, Paris, Université Paris 1.

Raymond R., 2004, Identifications sociales de la nature : représentations et arguments pour la gestion du territoire rural, Paris, Université Paris 1.

\section{NOTES}

1.Joëlle Salomon qui fait partie du Collectif approfondit tout particulièrement les «figures » des représentations anti-urbaines et leur effet sur l'aménagement du territoire en Suisse, en France et en Grande Bretagne (cf. Résumés de thèses dans ce volume) N. M.

2.Ces entretiens ont pour particularité 1) de rendre compte (selon un principe d'exactitude) du rapport entre la configuration matérielle des lieux « habités » et les discours se référant à ces mêmes lieux, qu'il s'agisse du « dedans » (plan de chaque pièce, mobilier, circulation, végétation et animaux, fenêtres et vues...) ou du dehors (l'environnement immédiat du lieu d'habitat de l'enquêté, mais aussi celui de ses déplacements et des lieux traversés situés par rapport à sa maison), en somme des valeurs qu'il accorde à chaque élément des lieux qui constitue sa « demeure » actuelle ; 2) de provoquer la narration de tous les lieux ayant valeur d'habitat dans le passé et dans le futur de l'enquêté, pour déboucher sur une mise en parallèle des qualités des lieux de vie les uns par rapport aux autres (d'où le nom de « récits de lieux de vie » ou de « biographie résidentielle ») ; 3) de tenter d'isoler dans le discours tout ce qui est connaissance de la matérialité et de la qualité des lieux évoqués ainsi que tout ce qui est de l'ordre des représentations et des « cultures de la nature ».

3.Florent Hebert a une formation d'architecte paysagiste. Outre l'approfondissement de cette piste de recherche " Y a-t-il un modèle masculin d'habiter ? », il apporte au Collectif sa réflexion sur l'habitabilité des formes « ordinaires », " banales », et pourtant très contraignantes, des constructions actuelles et anciennes ( $C f$. article dans ce numéro). Ses « récits de lieux de vie » confrontés à celles des autres répondront à ces questions. N. M. 
4.Premier point méritant approfondissement : est-ce que habiter dans le bien-être un chez soi, en réduisant l'écart entre le rêvé et l'aménagé, suffit pour induire ou engager des pratiques « durables » de tous les lieux de « résidence » ou de «traverse »? N. M. $5 . I l$ reste à démontrer qu'il s'agit bien d'un modèle féminin de " culture de la nature » impliquant une « conscience écologique » des lieux et milieux ; que ce modèle a valeur représentative, est partagé par d'autres familles d'intellectuels ou est observée dans d'autre groupes sociaux (Mathieu, Pilleboue, en cours) N. M.

6. Mais par qui cette « cohabitation » du vivant humain et du vivant animal et végétal est considérée « durable »? Y a-t-il partage possible de tous les espaces de l'intimité dans l'espace public ? L'appropriation du dehors engendre-t-elle des habitats publics soutenables? N. M.

7.Mais de quelle maîtrise s'agit-il ? L'extension déjà repérée chez la nourrisseuse implique-t-elle une gestion soutenable des lieux « maîtrisés »? N. M.

8.Quartier d'artisanat du meuble dès le $\mathrm{XVI}^{\mathrm{e}}$ siècle. Les artistes ont en partie succédé aux artisans dans les années soixante-dix-quatre-vingt. Désormais, nombre de locaux accueillent des activités de bureau.

9.La dégradation de l'habitabilité d'un milieu par un mode de partage de l'espace public qui contredit l'aspiration à étendre l'espace du chez soi au-delà du « foyer ", provoque des conflits et des frustrations, conduit-il à un repli sur des « espaces refuges » et, de ce fait, à une gestion insoutenable de ce milieu ? N. M.

10.Tiré d'Alain Vulic, 1991, Le cabaret le bistrot, lieu de sociabilité populaire dans le bassin houiller du Nord-Pas-de-Calais (1750-1985), Thèse d'histoire, Lille 3.

11. " Urbanité » doit être compris comme la manière d'être urbain, c'est-à-dire l'utilisation dominante par un individu de la ville, les ressources nécessaires à son épanouissement qu'il y puise, le comportement de chaque personne ne se réduisant pas à un de ces modèles.

\section{RÉSUMÉS}

Avec pour hypothèse commune que la montée actuelle de la "question habitante » dans les sociétés post industrielles oblige à mettre au centre de l'investigation : ${ }^{1}$. le point de vue de l'individu habitant, à partir duquel il faut tenter de définir les "cultures de la nature ", les représentations de la valeur des lieux, les catégories par lesquelles chaque sujet pense sa " géographicité »; 2. les catégories de rural et d'urbain rendent possible la mise en évidence des propriétés et des qualités de chaque milieu quel que soit le pays d'Europe concerné. Les auteurs tentent de dégager des résultats transversaux des récits de lieux de vie obtenus dans leurs terrains spécifiques de recherche. Ils aboutissent à une problématique collective et à une méthodologie à mettre en place pour approfondir ce qu'habiter veut dire aujourd'hui dans les représentations et les pratiques des ruraux et des urbains. La contradiction entre le chez soi pensé et pratiqué comme « espace refuge » ou «dedans » et le chez soi vu comme appropriation de l'abord et intégration de la discontinuité du dehors est un principe de distinction fort pour aller vers une typologie des « modes d'habiter ». 
The authors base their reflection on the common assumption that the growing importance of the "living issue" in post-industrial societies compels them to focus their investigation on: 1) the point of view of the resident, from which they must try to define the "cultures of nature", the representations of the value of the place and the categories through which each individual relates to his "geographicity"; 2) the categories of the rural and the urban whose confrontation is likely to evidence the qualities and properties of any European-based "milieu". The authors then synthetise the data provided by the narratives collected in their own fields of investigation. They can thus formulate a common question and methodology in order to reflect on what "living" means today in the representations and practices of country people and city dwellers. The contradiction between the home considered and experienced as a shelter or "inside" and the home seen as an appropriation of the margins and integration of the discontinuity of the "outside" is a strong distinctive principle to head towards a typology of the "ways of living".

INDEX

Mots-clés : Habiter

\section{AUTEURS}

\section{RICHARD RAYMOND}

UMR Ladyss, CNRS/Paris 1/Paris 8/Paris 10, mathieu@univ-paris1.fr 